\title{
A NOTE ON THE MODULUS OF CONVEXITY
}

\author{
by ANDREW T. PLANT
}

(Received 13 November, 1979)

In [1, Corollary 5], Figiel gives an elegant demonstration that the modulus of convexity $\delta$ in real Banach space $X$ is nondecreasing, where

$$
\begin{aligned}
\delta(\varepsilon) & =\inf \left\{1-\|x+y\| / 2: x, y \in S_{X},\|x-y\|=\varepsilon\right\}, \\
S_{X} & =\{x:\|x\|=1\}
\end{aligned}
$$

It is deduced from this that in fact $\delta(\varepsilon) / \varepsilon$ is nondecreasing [Proposition 3]. During the course of the proof [Lemma 4] it is stated that if $v \in S_{X}$ is a local maximum on $S_{X}$ of $\varphi \in S_{X^{*}}$, then $v$ is a global maximum $(\varphi(v)=1)$. This is false; it could be that $v$ is a global minimum. It is easy to construct such an example in $\mathbb{R}^{2}$ endowed with the maximum norm. What is true is that $v$ is a global maximum of $|\varphi|$.

To see this, choose $\varepsilon>0$ and $u \in S_{X}$ such that $\varphi(u)>1-\varepsilon$. Then, by local maximality, $\varphi((v+\lambda u) /\|v+\lambda u\|) \leqslant \varphi(v)$ for all sufficiently small positive $\lambda$. By rearrangement

$$
\lambda(1-\varepsilon)<\lambda \varphi(u) \leqslant(\|v+\lambda u\|-\|v\|) \varphi(v) \leqslant \lambda|\varphi(v)| .
$$

Hence, as required, $|\varphi(v)|=1$.

Making the necessary modifications to [1, Lemma 4], we complete this note by proving the following result.

LEMMA. $\delta(\varepsilon)=\inf \left\{1-\|x+y\| / 2: x, y \in B_{X},\|x-y\|=\varepsilon\right\}$, where $B_{X}=\{x:\|x\| \leqslant 1\}$.

Monotonicity of $\delta$ then follows easily as in Corollary 5 of [1].

Proof of Lemma. Without loss in generality we may assume $X$ is finite dimensional and $\varepsilon>0$. Let $x$ and $y$ be chosen in $B_{X}$ such that $\|x+y\|$ is maximal, subject to $\|x-y\|=\varepsilon$. Assume $\|x\| \leqslant\|y\|$. Then $y \neq 0$.

In fact $\|y\|=1$. To see this, set $x_{1}=(x-c(x-y)) /\|y\|$ and $y_{1}=(y+c(x-y)) /\|y\|$, where $c=(1-\|y\|) / 2$. Then $x_{1}, y_{1} \in B_{X},\left\|x_{1}-y_{1}\right\|=\varepsilon$ and $\left\|x_{1}+y_{1}\right\|=\|x+y\| /\|y\|$. Hence, by maximality of $\|x+y\|,\|y\|=1$.

To complete the proof we show either $\|x\|=1$, or $z \in B_{X} \cap\left(y+\varepsilon S_{X}\right)$ implies $\|z+y\|=$ $\|x+y\|$. Since $S_{X}$ is connected $(\operatorname{dim} X \geqslant 2), S_{X} \cap\left(y+\varepsilon S_{X}\right) \neq \varnothing$ for $\varepsilon \leqslant 2$. Hence the lemma holds whichever case applies.

Assume $\|x\|<1$. Choose $\varphi \in S_{X^{*}}$ such that $\varphi(x+y)=\|x+y\|$. Let $z \in B_{X} \cap\left(y+\varepsilon S_{X}\right)$. By maximality of $\|x+y\|$,

$$
\varphi(z+y) \leqslant\|z+y\| \leqslant\|x+y\|=\varphi(x+y) .
$$

So $\varphi(z) \leqslant \varphi(x)$, and hence, $(x-y) / \varepsilon$ is a local maximum of $\varphi$ on $S_{X}$. Either (a) $\varphi(x-y)=\|x-y\|$ or (b) $\varphi(x-y)=-\|x-y\|$.

Glasgow Math. J. 22 (1981) 157-158. 
If (a) holds,

$$
\begin{aligned}
1 & =\|y\| \leqslant(\|x+y\|+\|x-y\|) / 2 \\
& =(\varphi(x+y)+\varphi(x-y)) / 2=\varphi(x),
\end{aligned}
$$

implying $\|x\|=1$. Consequently, if $\|x\|<1$, (b) holds. Then $\varphi(z-y) \geqslant-\varepsilon=\varphi(x-y)$ and so, using (1), $\|z+y\|=\|x+y\|$, as required.

\section{REFERENCE}

1. T. Figiel, On the moduli of convexity and smoothness, Studia Math. 56 (1976), 121-155.

Department of Mathematics

UNIVERSITY GARDENS

GLASGOW G12 8QW 